BNL-73481-2005-CP

\title{
Beam-Beam Simulations Fur The eRHIC Electron Ring
}

\author{
C. Montag \\ Presented at the Particle Accelerator Conference(PAC'05) \\ Knoxville, Tennessee \\ May 16-20, 2005
}

\author{
Collider-Accelerator Department \\ Brookhaven National Laboratory \\ P.O. Box 5000 \\ Upton, NY 11973-5000 \\ www.bnlgov \\ Managed by \\ Brookhaven Science Associates, LLC \\ for the United States Department of Energy under \\ Contract No. DE-AC02-98CH10886
}

This is a preprint of a paper intended for publication in a journal $\propto$ proceedings. Since changes may be made before publication, this preprint is made availablewith the understanding that it will not be cited ar reproduced without the permission of the author. 


\section{DISCLAIMER}

This report was prepared as an account of work sponsored by an agency of the United States Government. Neither the United States Government nor any agency thereof, nor any of their employees, nor any of their contractors, subcontractors, or their employees, makes any warranty, express or implied, or assumes any legal liability or responsibility for the accuracy, completeness, or any third party's use or the results of such use of any information, apparatus, product, or process disclosed, or represents that its use would not infringe privately owned rights. Reference herein to any specific commercial product, process, or service by trade name, trademark, manufacturer, or otherwise, does not necessarily constitute or imply its endorsement, recommendation, or favoring by the United States Government or any agency thereof or its contractors or subcontractors. The views and opinions of authors expressed herein do not necessarily state or reflect those of the United States Government or any agency thereof.

FOR UNCLASSIFIED, UNLIMITED STIPRODUCTS

Available electronically at:

OSTI:

http://ww.osti.gov/bridge

Available for a processing fee to U.S. Department of Energy and its contractors, in paper from:

U.S. Department of Energy

Office of Scientific and Technical Information

P.O. Box 62

Oak Ridge, TN 37831

Phone: (865) 576-8401

Facsimile: (865) 576-5728

E-mail: reports@adonis.osti.gov

National Technical Information Service (NTIS):

Available for sale to the public from:

U.S. Department of Commerce

National Technical Information Service

5285 Port Royal Road

Springfield, VA 22131

Phone: (800) 553-6847

Facsimile: (703) 605-6900

Online ordering: http://www.ntis.gov/ondering.htm

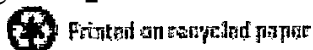




\title{
BEAM-BEAM SIMULATIONS FOR THE ERHIC ELECTRON RING *
}

\author{
Christoph Montag, Brookhaven National Laboratory, Upton, NY 11973, USA
}

\begin{abstract}
To study collisions between polarized electrons and heavy ions or polarized protons at high energy, adding a $10 \mathrm{GeV}$ electron storage ring to the existing RHIC facility is currently under consideration. To achieve high luminosities of several $10^{33} \mathrm{~cm}^{-2} \mathrm{sec}^{-1}$ range, a vertical beambeam tuneshift parameter of $\&=0.08$ is required for the electron beam. Simulation studies are being performed to study the feasibility of this high tuneshift parameter and explore the potential for even higher tuneshifts. Recent results of these studies are presented.
\end{abstract}

\section{INTRODUCTION}

The electron-ion collider eRHIC [1], currently under study at BNL, consists of a $10 \mathrm{GeV}$ electron ring added to the existing RHIC accelerator complex to study collisions of polarized electrons and relativistic heavy ions or polarized protons. The circumference of this electron ring is one third of the RHIC circumference. Some machine parameters of this facility are listed in Table 1.

A beam-beam tuneshift parameter of $\xi_{y}=0.08$ is required for the electron beam to achieve a luminosity of several $10^{32} \mathrm{~cm}^{-2} \mathrm{sec}^{-1}$. These high tuneshift parameters require careful simulation studies to ensure the feasibility of attaining the projected luminosity. Additionally, the unequal circumferences of the two rings lead to additional resonances that must be avoided when choosing the working point of the machine.

\section{UNEQUAL CIRCUMFERENCES}

Colliders comprised of storagerings of different circumference require careful choice of the working point of both rings to ensure stable beam operation [2]. The resonance condition is

$$
Q_{1}-\frac{C_{1}}{C_{2}} Q_{2}=n, \quad n \text { integer, }
$$

where $Q_{1}$ and $Q_{2}$ are the fractional tunes of the two machines and $C_{1}$ and $C_{2}$ denote their respective circumferences. The actual width of these resonances is most easily studied by simulations. In the case of eRHIC, $C_{1} / C_{2}=3$, so each electron bunch collides with three differenthadron bunches.

The simulation is performed by describing both beams as rigid Gaussian bunches. One electron bunch and three

\footnotetext{
*Workperformed underthe auspices of the U.S.Department of Energy
}

\begin{tabular}{lr}
\hline \hline electrons: & \\
ring circumference[m] & 1278 \\
number of bunches & 120 \\
geometric emittancehor./vert. [nm] & $53 / 9.5$ \\
$\beta$ functions hor//vert. [m] & $0.19 / 0.27$ \\
bunch length [mm] & 11.7 \\
synchrotrontune & 0.04 \\
particleshunch & $1.0 \cdot 10^{11}$ \\
beam-beam tune shifthor//vert. & $0.027 / 0.08$ \\
dampingtimes hor./vert./long. [turns] & $1740 / 1740 / 870$ \\
\hline hadrons: & \\
ring circumference[m] & 3834 \\
number of bun ches & 360 \\
geometric emittancehor./vert. [nm] & $9.5 / 9.5$ \\
$\beta$ functions hor./vert. [m] & $1.08 / 0.27$ \\
particles/bunch & $1.0 \cdot 10^{11}(\mathrm{p})$, \\
& $1.0 \cdot 10^{9}(\mathrm{Au})$ \\
beam-beam tune shifthor./vert. & $0.007 / 0.0035$ \\
\hline beam spot size hor.vert. [ $\mu \mathrm{m}]$ & $100 / 50$ \\
luminosity [cm ${ }^{-2}$ sec $^{-1}$ ] & $4.4 \cdot 10^{32}$ \\
\hline
\end{tabular}

Table 1: Interaction region parameters of the electron-ion collider ERHIC.

hadron bunches are simulated. The beam-beaminteraction is modelled as a mutual weak-strong kick, while the accelerator is described by a linear one-turn matrix. To seed the possible resonance, the electron bunch starts with an offset of $1 \mu \mathrm{m}$ at the interaction point (IP), small comparedto the rms beam size of $\sigma_{x}=100 \mu \mathrm{m}$ and $\sigma_{y}=50 \mathrm{pm}$. Bunches are tracked for $3 \cdot 10^{4}$ electron beam turns, correspondingto $10^{4}$ hadron beam turns in RHIC. Stability is defined as no increase of the electron beam amplitude during tracking.

Taking into accountboth planes and setting the ion beam tunes to the current RHIC working point $Q_{p, x}=.21$, $Q_{p, y}=.23$, a sufficientlylarge region of the electron beam tune space $.06<Q_{e, x}<.34, .05<Q_{e, y}<.28$ leads to stable motion, as shown in Figure 1.

\section{DYNAMIC FOCUSING}

With a beam-beam tuneshift parameter as high as $\xi_{y}=0.08$, the beam-beam interaction has a significant effect on the entire electron beam dynamics and cannot be treated as a small perturbation. For instance, the resulting small amplitudetune $\mathrm{Q}$ is expressed as

$$
\cos (2 \pi Q)=\cos \left(2 \pi Q_{0}\right)-2 \pi \xi \sin \left(2 \pi Q_{0}\right)
$$




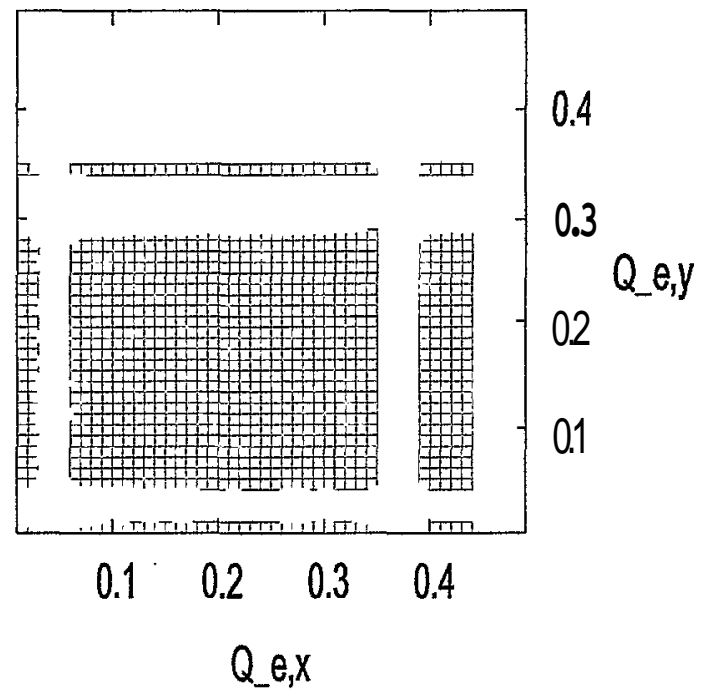

Figure 1: Electron beam tune diagram, $Q_{e, y}$ vs. $Q_{e, x}$, for a hadron working point of $Q_{p, x}=.21, Q_{p, y}=.23$. The checkered areas indicate stable tune regions.

Likewise, the strong beam-beam lens at the $\mathbb{P}$ significantly modifies the $\beta$-function at the IP, resulting in a tunedependent $\beta$-function,

$$
\beta=\frac{\beta_{0}}{\sqrt{1+4 \pi \xi \cot \left(2 \pi Q_{0}\right)-4 \pi^{2} \xi^{2}}} .
$$

The resulting $\beta$-function at the IP is therefore significantly reduced for tunesjust above the integer, which provides additional focusing.

The presence of this strong beam-beam lens also modifies the entire machine optics and therefore the equilibrium beam emittance, which depends on the "curly H" function

$$
\mathcal{H}(s)=\beta(s){D^{\prime}}^{2}(s)+2 \alpha(s) D(s) D^{\prime}(s)+\gamma(s) D^{2}(s),
$$

where $\alpha(s), \beta(s)$, and $\dot{\gamma}(s)$ are the Twiss parameters at location $s, D(s)$ is the dispersionin the same location, and $D^{\prime}(s)=\mathrm{d} D(s) / \mathrm{d} s$. The resulting dynamic emittance $E$ can be approximatedas [3]

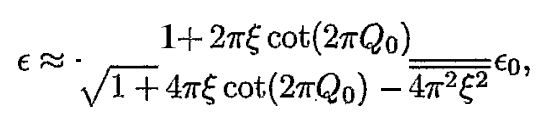

where $\epsilon_{0}$ refers to the equilibrium emittance of the unperturbed lattice, but in fact dependsrather strongly on the machine lattice [4]. FOP the simulation, the resulting equilibrium emittance is therefore calculated with the respective actual dynamic machine lattice for each working point.

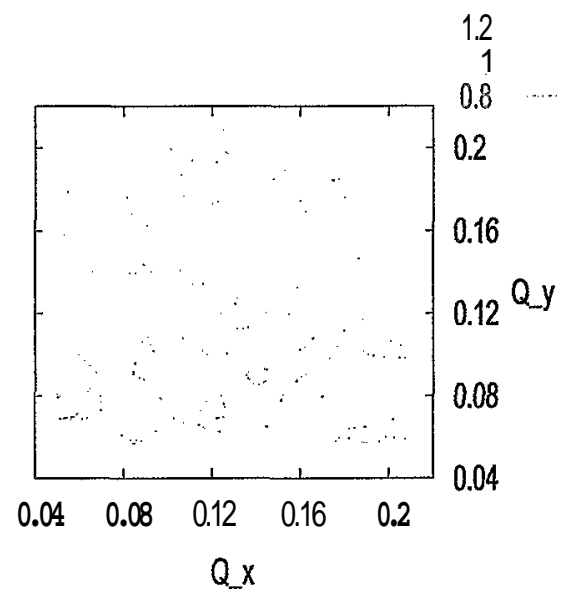

Figure 2: Contourplot of the resulting normalized luminosity $\mathcal{L} / \mathcal{L}_{0}$ vs. horizontal and vertical electronbeam tune. $\mathcal{L}_{0}$ denotesthe geometric design luminosity accordingto Table 1.

\section{SIMULATIONRESULTS}

The nonlinear eRHIC electron ring lattice [1] is optimized for a working point of $Q_{x}=.10, \mathrm{Q}=.14$ and zero chromaticity in both planes. Tracking studies were performed to find the best working point for this machine, scanning tunes in the range between the integer and the quarter resonance by adjusting the main quadrupoles accordingly. For each working point the chromaticities are readjusted to zero.

The beam is represented by 100 macroparticles with a rms momentum deviation of $\sigma_{p}=0.007$ and a synchrotrontune of $Q_{s}=\mathbf{0 . 0 4}$. These particles are tracked for ten radiation dampingtimes, including quantum excitation and radiation damping. The horizontal equilibriumemittance is adjusted according to the radiation integrals that correspond to each individual working point. The vertical equilibrium emittance is assumed to be unaffectedby the machinetune. The resulting equilibriumluminosity is calculated according to the obtained rms beam sizes $\sigma_{x}$ and $\sigma_{y}$ after tracking for ten damping times. Figure 2 shows a contour plot of the resulting luminosity in units of the nominal geometric luminosity, as a function of the working point $\left(Q_{x}, \mathrm{Q}\right)$.

While the luminosity generally increaseswith lower tunes, the coupling resonance is clearly visible in this plot, as is the 6th order resonance in both planes. At very low tunes synchrotronsidebandsenhancethe width of the integer resonance. However, to ensure proper matching of beam sizes of the hadron and electron beam at the $\mathrm{P}$, both planes have to be checked separately. Figures 3 and 4 depict the result- 


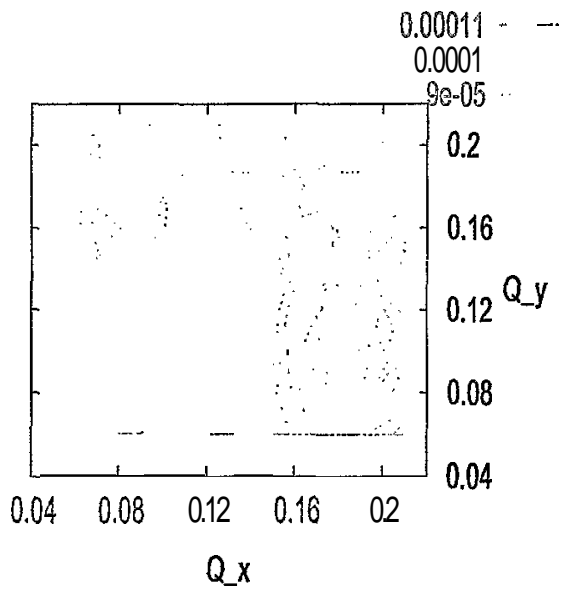

Figure 3: Contour plot of the horizontal rms electron beam size $\sigma_{x}$ (in meters) vs. electron beam working point $\left(Q_{x}, Q_{y}\right)$.

ing rms beam sizes in the two planes as a function of the working point. In the horizontal plane, a significant beam size increase occurs mostly along the 6 th order resonance line. The situation in the vertical plane is more complex due to the larger beam-beam parameter.

\section{CONCLUSION}

Simulation studies show that even with beam-beam tuneshift parameters of up to $\xi=0.08$, sufficiently large areas in the working diagram can be found that support the projected luminosity performance of $\mathrm{RHIC}$. These simulations also indicate that a luminosity significantly higher than the geometrical one could be achieved by moving the electron beam tunes very close to the integer, thus taking advantage of dynamic focusing effects. However, this results in a beam size mismatch of the two beams, which may cause emittancedeterioration of the ion beam.

Based on these simulations three possible working points have been identified that promise to deliver the design luminosity without a significant beam size mismatch in either plane. These working points are around $\left(Q_{x}, Q_{y}\right)=$ $(.05, .07),(.10, .14)$, and $(.14, .07)$. These working points are further investigated in terms of non-Gaussian tails resulting from the beam-beaminteraction in conjunctionwith quantum excitation and radiation damping [5].

According to simulations, unequal circumferences of the two rings are not much of a concern in terms of barycenter motion of the two beams, as long as certain additional resonances are avoided. The remaining stable tune space is

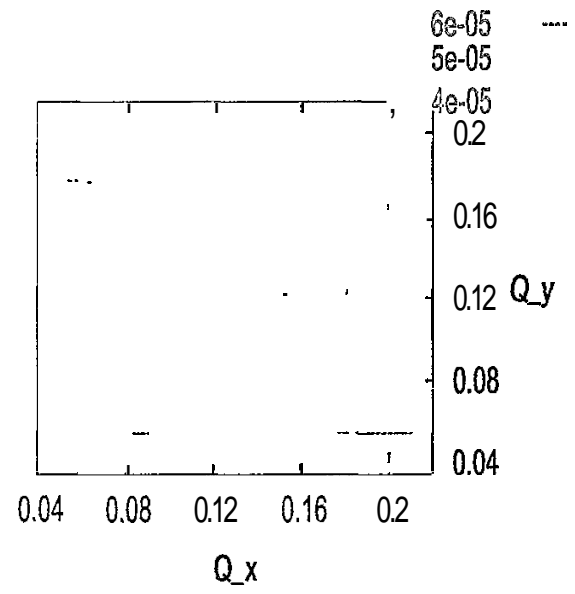

Figure 4: Contour plot of the vertical rms electron beam size $\sigma_{y}$ (in meters) vs. electron beam working point $\left(Q_{x}, Q_{y}\right)$.

sufficiently large to ensure stable operation of the electronion collider eRHIC. The location of the stable area within the tune diagram depends on the working point chosen for the ion ring. For the present RHIC working point, stable electron tunes are consistent with those found necessary to achieve design luminosity.

\section{REFERENCES}

[1] eRHIC Zeroth-Order Design Report, BNL note C-A/AP/142

[2] K. Hirata and E. Keil, "Barycenter motion of beams due to beam-beam interaction in asymmetric colliders", Nucl. Instr. and Meth. A292 (1990) 156-168

[3] K. Firca, "Ekam-beam effects", in: A. W. Chao and M. Tigner (eds.), Handbook of Accelerator Physics and Engineering, World Scientific, Singapore(1999)

[4] A. V. Otboyev and E. A. Perevendentsev, "Self-consistent $\beta$ functions and emittances of round colliding beams", Phys. Rev. ST Accel. Beams 2,104401 (1999)

[5] C. Montag, "Simulation of resonance streaming at the eRHIC electron storagering", these proceedings 